\title{
INHIBITION CONTROL AND WORKING MEMORY CAPACITY IN CHILDREN WITH SLI
}

\author{
Klara MARTON ${ }^{122}$, Lyudmyla KELMENSON'), and Milana PINKHASOVA ${ }^{1)}$ \\ ${ }^{1)}$ City University of New York, U.S.A., ${ }^{2)}$ Eotvos Lorand University, Hungary
}

\begin{abstract}
This study examined the "inefficient inhibition hypothesis" (IIH; Bjorklund \& Harnishfeger, 1990; Wilson \& Kipp, 1998) in three groups: children with specific language impairment (SLI), age-matched and language-matched controls. The IIH suggests that individuals with efficient inhibition skills perform better on working memory tasks because they are able to keep out irrelevant information from working memory. Children with SLI show processing capacity limitations. This study examined whether the working memory limitations are impacted by inhibition problems in this population. Working memory capacity was measured with a listening span task and children's inhibition errors were categorized. These errors reflected either immediate or delayed inhibition problems and they indicated either contextual distractions or perseverations. Children with SLI produced more inhibition errors than their peers in most categories. The results show an association between inhibition control and working memory capacity, but the direction of causality is not clear.
\end{abstract}

Key words: specific language impairment, inhibition control, working memory capacity

The aim of the present study was to examine the relationship between inhibition control and working memory. Specifically, this study evaluated the "inefficient inhibition hypothesis" (IIH; Bjorklund \& Harnishfeger, 1990; Wilson \& Kipp, 1998) in children with specific language impairment (SLI). This hypothesis suggests that individuals with better inhibition skills have more processing capacity than people who are less efficient in inhibition because the individuals in the former group are able to keep out irrelevant information from their memory. There is a general consensus in the literature that children with SLI have processing capacity limitations (Adams \& Gathercole, 2000; Ellis-Weismer \& Evans, 2002). The nature of this limitation, however, is not clear. If children with SLI show more difficulty in inhibition control than children with typical language development (TLD), then, according to the IIH, their processing capacity limitation is influenced by their poor inhibition control.

This study was supported by a research grant from the National Institute on Deafness and Other Communication Disorders (Working memory capacity in children with SLI, R03DC41449) and by a sabbatical year fellowship for the first author from Brooklyn College. We thank Sabina Ajani for her help in data coding and analysis. Special thanks to Michael Bergen for his continuous support in recruiting participants and for providing testing space in the Speech and Hearing Center of Brooklyn College.

Correspondence concerning this article should be addressed to Klara Marton, Ph.D., Brooklyn College, 2900 Bedford Avenue, Brooklyn, NY 11210 (email: kmarton@brooklyn.cuny.edu). 
Inhibition control may occur at both behavioral and cognitive levels. Behavioral level studies typically involve inhibition of motor responses and impulse control (e.g., Johannes et al., 2001). Cognitive level studies focus on the mental processes of attention and memory in contexts where unwanted thoughts or inappropriate meanings of ambiguous words have to be suppressed (e.g., Copland, Chenery, \& Murdoch, 2000; Gernsbacher \& Faust, 1991; Wegner, 1992; Wenzlaff \& Wegner, 2000).

Inhibition control plays a major role in tasks that involve temporal delays (Barkley, 1997; Friedman \& Miyake, 2004). It can prevent perseveration in task performance through the suppression of irrelevant information. The frequency of perseveration may be affected by task complexity, experimental conditions, and response modality (Stedron, Sahni, \& Munakata, 2005). In a latent variable analysis Friedman \& Miyake (2004) distinguished among three inhibition-related functions: 1. inhibition of a prepotent response, 2. resistance to distractor interference, 3. resistance to proactive interference. These inhibition functions are separate but closely related mechanisms that also have a strong relationship with working memory.

Inhibition of a prepotent response refers to the ability to suppress a dominant response. During task performance, when there is a delay in response, the contents of working memory have to be protected from external interfering stimuli that can distort or disrupt them. Resistance to distractor interference refers to this function. Resistance to distractor interference has often been associated with focused attention (Melara, Rao, \& Tong, 2002; Friedman \& Miyake, 2004) because the attentional system governs dual excitatory and inhibitory processes. These processes work together to protect the relevant contents of working memory from the influence of irrelevant, but interfering external stimuli. Resistance to distractor interference and proactive interference both involve interference control mechanisms. The latter function, however, refers to the ability to resist memory intrusions from previously, but no longer relevant items.

Wilson and Kipp (1998) define the functions of inhibition and resistance to interference somewhat differently than Friedman and Miyake (2004). According to Wilson and Kipp, inhibition of a response involves an active suppression process, resistance to interference occurs passively. Encoding is a critical distinguishing feature between inhibition and resistance to interference. When inhibiting a response, we first encode the information into working memory, then determine that it is not relevant, and then expel the culprit from working memory. In contrast, resistance to interference occurs before the interfering item could enter working memory, thus, the interfering item is not encoded. Wilson and Kipp provided evidence for this distinguishing feature through experiments including recall and recognition memory tasks. Inhibited items cannot be recalled, but - because they have been encoded - they can be recognized. Interfering items, on the other hand, that have been resisted before entering into working memory cannot be recognized. Thus, only inhibition of a response interacts with the concept of limited cognitive resources - resistance to interference does not. Yet, there are similarities between inhibition of a response and resistance to interference. Both functions are gating mechanisms that develop with age. Their developmental patterns, however, differ: response inhibition becomes mature much earlier than resistance to interference. While 
interference control continues to develop through around 12 years of age, response inhibition develops rapidly during the preschool years (Bjorklund \& Harnishfeger, 1990). This study focuses on the question whether children with SLI are able to suppress information that had been encoded, but is not relevant anymore.

\section{Working memory and executive functions in children with SLI}

Children with SLI have deficits in working memory, in speech perception, and in processing speed, in addition to their well-documented deficits in morphosyntax, syntax, lexical semantics, and discourse (Leonard, 1998). Researchers have pointed to more general deficits, whether domain specific to language or domain general that may better explain the deficit pattern(s) in these children. Among such proposals have been notions that children with SLI have more limited processing capacity than children with TLD (Bishop, 1997). The literature on working memory in children with SLI is replete with investigations of phonological working memory (see for a review Montgomery, 2003), but few studies have directly investigated more complex functions including inhibition.

Inhibition control plays an important role in staying focused and constraining the contents of memory. This mechanism helps individuals to be goal-relevant (Hasher, Lustig, \& Zacks, 2007). Our previous results indicated that children with SLI show difficulty in simultaneous processing (Marton, 2006; Marton, Schwartz, Farkas, \& Katsnelson, 2006). Children with SLI showed diminished primacy and recency effects in listening span tasks. These children showed a weakness in processing the new incoming stimuli while rehearsing the old information simultaneously. There was no significant difference in the number of recalled stimuli across item positions for these children. Only $23 \%$ of the children with SLI showed primacy and recency effects. In contrast, primacy and recency effects were clear in the recall of most typically developing children. There are various interpretations of these data (e.g., problems in attention switching), however, one possibility is that the diminished primacy and recency effects reflect a weakness in inhibition control in children with SLI. Often these children either repeated the sentencefinal words or answered the questions that targeted the sentence content in the listening span tasks; they were not able to perform both operations concurrently. Their difficulties were not simply related to limitations in storage. Although these children remembered relatively few of the sentence-final words during testing, if testing was stopped and they were asked whether they could repeat the stimuli they were presented with, most children repeated entire sentences correctly. These children showed a deficit in repeating words in interfering contexts. The answers to the questions prior word repetition interfered with the words to-be-remembered.

Gillam, Cowan, and Day (1995) also reported problems with suppressing irrelevant information in children with SLI. In their study, children with SLI showed larger suffix effects than their peers. The suffix is an item that is added after the list-final item, therefore it diminishes the recency effect. Children with SLI produced more interference errors with the suffix than the control group because they did not suppress the irrelevant information.

Further, the results of various attention tasks also showed difficulty in focusing on 
the relevant information in children with SLI, particularly if the task was more demanding on working memory (Noterdaeme, Amorosa, Mildenberger, Sitter, \& Minow, 2001). There were no differences between the children with SLI and their TLD peers on simple vigilance tasks. The groups performed comparably on attention tasks with low working memory load. In a sustained visual attention task with higher working memory demands, however, the children with SLI made more errors than did the children in the control group. The authors concluded that selective and sustained attention are particularly impaired in children with SLI when the tasks have high working memory demands. Moreover, these findings indicate a weakness in inhibition control in children with SLI because it is crucial to overcome the influence of irrelevant stimuli on relevant context in sustained attention tasks (Melara et al., 2002).

Working memory tasks involving simultaneous processing are highly demanding on inhibition control. Although children with SLI perform more poorly in these tasks than their age-matched peers, inhibition control and its impact on working memory is an uncharted area of SLI studies. This paper is part of a larger study that targets the relations among working memory, executive functions, and long-term memory. In this project we examined working memory capacity of children with typical and atypical language development and performed a detailed error analysis to test the IIH in two respects. It was hypothesized that children with SLI show limitations in a listening span task that measures processing capacity. Although there is a large amount of evidence in the literature for SLI children's working memory capacity limitations, we tested this hypothesis to ensure that the SLI participants of the present study performed similarly to reported data. The second hypothesis was that children with more limited working memory capacity indicate more difficulty in suppressing irrelevant information than children with better processing capacity, as suggested by the IIH. The third hypothesis was that an increase in set size the number of stimuli to remember- results in a decrease in working memory performance accuracy and in an increase in inhibition errors.

\section{METHODS}

\section{Participants}

Three groups of children (15 children with SLI, 15 age-matched peers, and 15 language-matched controls) participated in this study. All participants attended elementary school and used English as their primary language. The first group consisted of children with SLI (age-range: 7; 8-10; 1 years; mean age: 8; 9) who had been diagnosed by a speech-language pathologist as having specific language impairment. All children in this group were enrolled in speech-language treatment at the time of the study. Their language performance was at least 1.25 SD below the age appropriate level (range: $81-64$ ) as measured by the Clinical Evaluation of Language Fundamentals-4 (CELF-4) language test (Semel, Wiig, \& Secord, 2003). All participants in this group showed typical $(>90)$ nonverbal intelligence on the Test of Nonverbal Intelligence (TONI-3, Brown, Sherbenon, Johnsen, 1997).

The second group included chronological age-matched (within 3 months) typically developing children (AM), ranging in age between 7; 9 and 10;3 years. Interviews with parents and teachers ensured that all children in this group followed a typical developmental pattern. These participants passed a language screening (CELF-3 Screening Test; Semel, Wiig, \& Secord, 1995) and showed typical nonverbal IQ (see more details in Table1). 
Table 1. Participant profile

\begin{tabular}{ccccc}
\hline & $\begin{array}{c}\text { Age } \\
\text { (years) }\end{array}$ & $\begin{array}{c}\text { CELF-4 standard score/CELF-3 } \\
\text { SCREENING criterion score* }\end{array}$ & $\begin{array}{c}\text { PPVT } \\
\text { standard score }\end{array}$ & $\begin{array}{c}\text { TONI } \\
\text { IQ }\end{array}$ \\
\hline SLI & & & & \\
mean & 8.94 & 72.53 & 83.27 & 104.47 \\
$S D$ & 0.9 & 9.26 & 11.42 & 17.12 \\
AM & & & & \\
mean & 8.82 & 25.71 & 99 & 110 \\
SD & 0.93 & 5.59 & 8.7 & 16.59 \\
LM & & & & 110.6 \\
mean & 7.52 & 20.93 & 95.8 & 16.65 \\
SD & 0.71 & 13.09 & 17.45 & 10 \\
\hline
\end{tabular}

* All participants in the SLI group were tested with the CELF-4 comprehensive language test. Table 1 shows their mean standard score. The 2 control groups passed a language screening with the CELF-3 Screening test. This test provides criterion scores. Table 1 shows the mean criterion scores for the AM and LM groups.

The third group consisted of language-matched typically developing children who were younger than the participants in the other 2 groups. Each child in this group was matched to 1 of the children with SLI within 3 raw scores on the Peabody Picture Vocabulary Test-III (Dunn \& Dunn, 1997). Similar to the agematched participants, these children also passed a language screening (CELF-3 Screening Test) and exhibited typical nonverbal IQ (see Table 1).

There were 2 girls and 13 boys in each group. All participants passed a pure-tone audiometric screening of both ears at $20 \mathrm{~dB} \mathrm{HL}$ (at 500, 1000, 2000, and $4000 \mathrm{~Hz}$ ). None of the children demonstrated articulatory errors, motor, emotional, or physical handicaps.

\section{Stimuli}

Listening Span Task

The task included 2 lists of sentences ( 3 sets of 2 sentences per set, 3 sets of 3 sentences per set, 3 sets of 4 sentences per set, and 3 sets of 5 sentences per set in each list; total of 84 sentences). There was a question targeting sentence content in each set. Participants listened to the sentences, answered the question following sentence presentation, and then repeated the sentence-final word. The sentences were short including 10 or fewer syllables (see sample sentences in the Appendix). The sentence-final words were high frequency words that children typically acquire during their preschool years, and they had been controlled for their phonological features (e.g., they were all 1-2-syllable words). Frequency ratings were based on data from Hall, Nagy, \& Linn (1984). The task was designed to test processing capacity/simultaneous processing and storage.

\section{Procedures}

As mentioned above, this research is part of a larger study that examines the relationship among working memory, executive functions, and the long-term lexicon (Marton, 2006; Marton, Schwartz, Kelmenson, \& Pinkhasova, in preparation). Participants were tested individually in a single session in the Speech and Hearing Center at Brooklyn College. All stimuli were audio recorded by a female speaker. The participants listened to the stimuli through headphones and their responses were tape-recorded.

The sentence sets of the listening span task were presented in a random order. Participants were asked to listen to a set at a time and repeat the sentence-final words of that set in the same order as they were presented. Each set included a question to ensure that children processed the entire sentence, not only the 
sentence-final word. Participants were instructed to answer first the question and then repeat the sentencefinal words. The answer to the question was never a sentence-final word. Practice trials with different stimuli preceded testing.

\section{Data analysis}

Different investigators performed testing and data analysis for each child. An answer was considered as an inhibition error if it was a target of a previous list or a non sentence-final word. Inhibition errors were categorized as immediate (from the current set) or delayed (from a previous set) and as contextual distractions or perseverations. Immediate errors were non-target items from the current set, whereas delayed errors were non-target items from a previous set. Contextual distractions were errors where the child recalled a nontarget word from the middle of the sentence or from the question, whereas perseverations included errors where the child repeated either a previously recalled sentence-final word or the answer to the question (see examples for errors in the Appendix).

Interjudge reliability of error coding was calculated on 172 randomly chosen sentences/responses from all participants. The two researchers provided identical transcriptions for 171 sentences.

\section{RESULTS}

The first hypothesis of this study, children with SLI have working memory capacity limitations, was tested with the calculation of children's listening span. A larger listening span was predicted in the AM and LM groups than in the group of children with SLI. Pairwise comparisons showed the following group effects: SLI-AM: $F(1,27)=17.04$, $p<0.001 ; d: 1.5$, SLI-LM: $F(1,27)=5.84, p<0.05$; $d: 0.87$; AM-LM: $F(1,27)=1.92$, $p=0.18 ; d: 0.52$. Children with SLI performed more poorly than their age-matched and language-matched peers. The control groups did not differ from each other.

Further, it was hypothesized that an increase in set size (2-3-4-5 sentences per set) results in a decrease in performance accuracy in all groups. Factorial ANOVA indicated a main effect for group $(F(2,168)=28.19, p<0.01$; SLI-AM: $d: 1.22$, SLI-LM: $d: 0.37$, AM-LM: $d: 0.72)$ and a main effect for set size $(F(3,168)=25.39, p<0.001 ; 2-3: d: 1.1$, $3-4: d: 0.3,4-5: d: 0.33)$. There was no group $\times$ set size interaction $(F(6,168)=0.55$, $p=0.77)$. All children's performance accuracy was negatively affected by an increase in set size (see Table 3).

The second hypothesis of this study was that children with limited working memory capacity produce more inhibition errors than children with more working memory capacity. The results showed group differences in the frequency of inhibition errors: SLIAM: $F(1,28)=9.9, p<0.01 ; d: 1.15$; SLI-LM: $F(1,28)=10.89, p<0.01 ; d: 1.2$, AMLM: $F(1,28)=0.08, p=0.78 ; d: 0.1$. Children with SLI produced significantly more inhibition errors than the typically developing children. Participants of the AM and LM groups did not differ in the number of inhibition errors they produced.

Inhibition errors were further analyzed whether they reflected immediate distraction or a delayed intrusion. A factorial ANOVA revealed a main effect for both independent variables and there was a group $\times$ error type interaction (group effect: $F(2,84)=8.63$, $p<0.001$; inhibition type: $F(1,84)=75.58, p<0.001$; interaction: $F(2,84)=5.97$, $p<0.01)$. Within group comparisons indicated that all children produced more immediate than delayed errors (immediate-delayed errors within the SLI group: $F(1,28)=35.62$, 
Table 2. Working memory capacity and inhibition errors

\begin{tabular}{cccccc}
\hline & Listening span & Immediate errors & Delayed errors & Contextual distraction & Perseveration \\
\hline SLI & & & & & \\
mean & 1.76 & 26.44 & 1.83 & 16.4 & 11.87 \\
$S D$ & 0.74 & 11.31 & 0.98 & 9.72 & 10.74 \\
AM & & & & & \\
mean & 3.14 & 11.85 & 1.22 & 8 & 5.07 \\
SD & 1.23 & 7.65 & 0.62 & 6.8 & 4.44 \\
LM & & & & & \\
mean & 2.5 & 10.81 & 1.12 & 7.2 & 4.73 \\
SD & 1.22 & 6.47 & 0.52 & 4.16 & 3.9 \\
\hline
\end{tabular}

$p<0.001 ; d: 2.18$, within the AM group: $F(1,28)=12.52, p<0.01 ; d: 1.29$, within the LM group: $F(1,28)=34.06, p<0.001 ; d: 2.13)$. Participants in the SLI group differed from the AM and LM groups in the amount of immediate errors. These children produced more immediate inhibition errors than the typically developing children (SLI-AM: $d: 1.15$, SLI-LM: $d: 1.07)$. The age-matched and language-matched controls did not differ in the number of immediate inhibition errors (AM-LM: $d: 0.21$ ). There was no difference across groups in the number of delayed inhibition errors (see Table 2).

Inhibition errors were further categorized whether they reflected contextual distraction or perseveration. The results of factorial ANOVA showed a main effect for group $(F(2,83)=11.01, p<0.001)$ and a main effect for error type $(F(1,83)=4.33$, $p<0.05)$. There was no group $\times$ error type interaction $(F(2,83)=0.15, p=0.85)$. Children with SLI produced more errors in both categories than the typically developing children (SLI-AM contextual distraction: $d: 0.9$, SLI-LM: $d: 0.88$, SLI-AM perseveration: $d: 0.83$, SLI-LM: $d: 1.23)$. The two typically developing groups showed similar performance (AM-LM contextual distraction: $d: 0.11$, AM-LM perseveration: $d: 0.08$ ). All participants produced more contextual distraction errors than perseverations (see figure 1).

Factorial ANOVA was performed to assess hypothesis 3 that an increase in set size results in an increase in inhibition errors. There was a main effect for group $(F(2$, $168)=18.52$, SLI-AM: $d: 0.86$, SLI-LM: $d: 1.02$, AM-LM: $d: 0.07)$, but the increase in working memory load (set size) did not result in an increase in inhibition errors $(F(3$, $168)=2.35, p=0.075,2-3: d: 0.5,3-4: d: 0.08,4-5: d: 0.1)$. There seems to be a tendency, but this result did not reach significance (see Table 3 ). There was no group $\times$ set size interaction $(F(6,168)=0.54, p=0.9)$. 


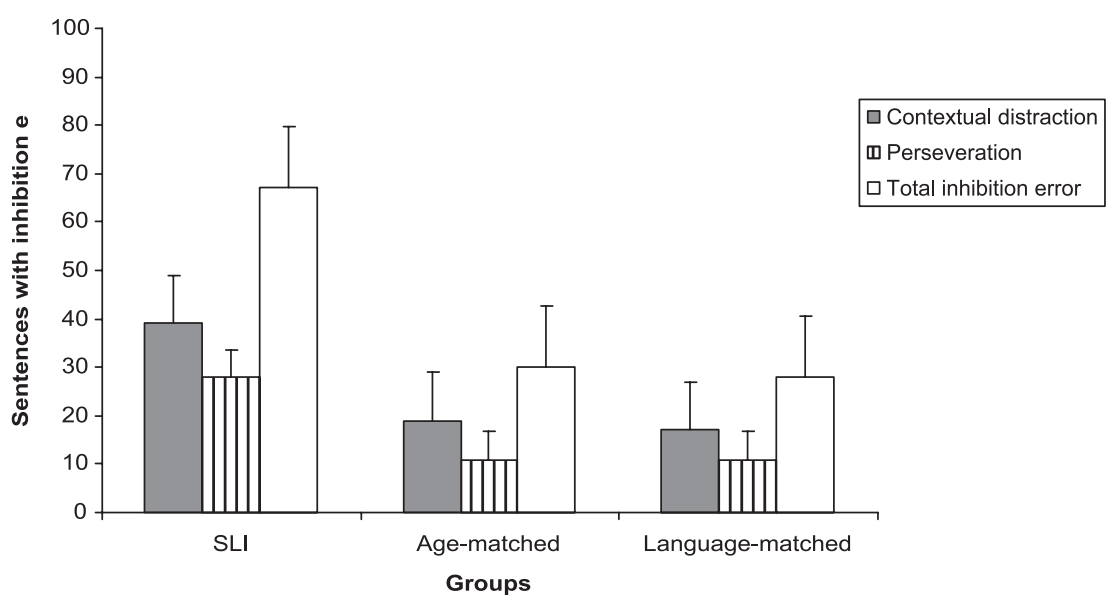

Fig. 1. Inhibition error types: Percentage of all sentences produced with an inhibition error

Table 3. The effect of working memory load on performance accuracy and on inhibition errors

\begin{tabular}{|c|c|c|c|c|c|c|c|c|}
\hline & \multicolumn{2}{|c|}{ Set of 2 sentences } & \multicolumn{2}{|c|}{ Set of 3} & \multicolumn{2}{|c|}{ Set of 4} & \multicolumn{2}{|c|}{ Set of 5} \\
\hline & $\begin{array}{c}\% \\
\text { correct }\end{array}$ & $\begin{array}{c}\text { Inhibition } \\
\text { errors }\end{array}$ & $\begin{array}{c}\% \\
\text { correct }\end{array}$ & $\begin{array}{l}\text { Inhibition } \\
\text { errors }\end{array}$ & $\begin{array}{c}\% \\
\text { correct }\end{array}$ & $\begin{array}{l}\text { Inhibition } \\
\text { errors }\end{array}$ & $\begin{array}{c}\% \\
\text { correct }\end{array}$ & $\begin{array}{c}\text { Inhibition } \\
\text { errors }\end{array}$ \\
\hline \multicolumn{9}{|l|}{ SLI } \\
\hline mean & 56.66 & 4.73 & 31.66 & 7 & 25.28 & 8.13 & 24.61 & 8.4 \\
\hline$S D$ & 20.34 & 3.06 & 12.1 & 4.83 & 13.75 & 5.94 & 11.1 & 6.85 \\
\hline \multicolumn{9}{|l|}{$\mathrm{AM}$} \\
\hline mean & 83.06 & 1.8 & 59.26 & 3.27 & 53.61 & 3.6 & 44.55 & 4.13 \\
\hline$S D$ & 10.62 & 1.68 & 18 & 2.4 & 20.33 & 3.2 & 19.27 & 3.41 \\
\hline \multicolumn{9}{|l|}{ LM } \\
\hline mean & 58.05 & 2.73 & 44.44 & 3.2 & 39.44 & 2.67 & 29.44 & 3.33 \\
\hline$S D$ & 25.9 & 2.31 & 27.03 & 2.14 & 23.3 & 2.26 & 17.61 & 2.58 \\
\hline
\end{tabular}

\section{DISCUSSION}

The aim of this study was to test the "inefficient inhibition hypothesis" (Bjorklund \& Harnishfeger, 1990; Wilson \& Kipp, 1998) in children with SLI. It has been widely accepted in the working memory literature that children's performance is influenced not only by domain-specific storage capacities, but also by domain-general processes (Bayliss, Jarrold, Gunn, \& Baddeley, 2003). The IIH suggests that individuals with efficient inhibition skills perform better on various working memory tasks because they are able to keep out irrelevant information from working memory. If subjects are able to 
expel previously relevant, but currently irrelevant information from working memory, then they have less material to-be-processed. Limited amount of material requires less processing space and capacity. Thus, working memory capacity can be enhanced by increasing the efficiency of inhibition control. Children with SLI are typically reported to have limitations in processing capacity. Thus, they are an excellent target group to test the IIH.

Hypothesis 1 predicted that children with SLI show decrements in listening span compared to their age-matched and language-matched peers. The listening span measures served to ensure that the SLI participants in this study show similar performance patterns to other reported data (e.g., Ellis-Weismer, Evans, \& Hesketh, 1999, Marton \& Schwartz, 2003, Marton et al., 2006; Montgomery, 1995, 2000). The results of the listening span task are in line with previous findings. Children with SLI performed more poorly than their age-matched and language-matched peers. There was an age-effect between the agematched and the language-matched groups in listening span. Yet despite the overall ageeffect, the two groups showed very similar performance patterns in terms of error types.

The second hypothesis was that children with SLI produce more inhibition errors than their peers. The results show a group effect in almost every aspect of inhibition control. Children with SLI produced more immediate inhibition errors than the controls. These children produced words that were previously relevant, but then had to be suppressed. There was no group difference in delayed inhibition. Every child showed more difficulty with suppressing recent items than with items from earlier sets. Children with SLI also repeated more words that reflected contextual distraction than their peers. These words were not sentence-final words. They had to be encoded during sentence processing, but they were not target items. These errors indicate that children with SLI have difficulty with expelling previously activated information from working memory when that information is not relevant anymore. This finding is similar to the results of Cornoldi et al. (2001). The authors studied working memory in children with attention deficit disorders. These children also showed significant interference between relevant information and information that first had to be processed, and subsequently excluded from memory.

Children with SLI in the present study produced more perseverations than the controls. Most of the perseverations were repetitions of the answer to the questions. Those answers were not sentence-final words. Following a number of perseverations, the investigators asked the children whether they remembered the instructions (that they were asked to repeat only the sentence-final words). All children with SLI were able to repeat the instructions correctly. Their problem was not related to remembering the instructions, but to suppressing irrelevant information. Our findings are in agreement with the results of previous research that examined inhibition in children with autism and used children with SLI as a control group (Bishop \& Norbury, 2005; Liss et al., 2001). These authors found poor inhibition in children with SLI compared to typically developing children on different neuropsychological tests.

Inhibition efficiency may be affected by various components, such as testing conditions, stimulus type, etc. This study examined the effect of memory load (an 
increase in set size) on performance accuracy and on the number of inhibition errors. Increased set size had a negative effect on all children's performance accuracy. All participants made more errors in recall as the number of sentence-final words increased. The effect of memory load on the number of inhibition errors is less clear. Typically developing children produced only a few inhibition errors, most of their errors were omissions, similar to other reported findings (McCormack, Brown, \& Vousden, 2000). Children with SLI showed a non-significant tendency for an increased number of inhibitions with an increase in memory load. Further examination of this question with more power is needed.

Although we found an association between inhibition control and working memory capacity, the direction of causality is not clear. More direct examinations of various inhibition functions should be performed in future research. Inhibition control is not limited to the suppression of irrelevant information. The evaluation of resistance to interference in children with SLI may help to clarify the relations between inhibition and working memory capacity. If resistance to interference works efficiently, irrelevant items are not encoded into working memory. The present study focused only on items that had been encoded previously. Thus, these data show that children with SLI have difficulty with suppressing information that is not relevant anymore. They show problems with expelling unnecessary material from working memory. These findings support the IIH, but more research is needed to understand SLI children's inhibition functions and their impact on working memory performance. The identification of weak inhibition functions in children with SLI will support clinicians and teachers in developing more appropriate therapeutic methods to help these children to remember and follow complex instructions and to perform in multilevel tasks that are the everyday, bread-and-butter requirements in any school settings.

\section{REFERENCES}

Adams, A-M., \& Gathercole, S. E. 2000. Limitations in working memory: implications for language development. International Journal of Language and Communication Disorders, 35, 95-116.

Bayliss, D. M., Jarrold, C., Gunn, M. D., \& Baddeley, A. D. 2003. The complexities of complex span: Explaining individual differences in working memory in children and adults. Journal of Experimental Psychology: General, 132, 71-92.

Barkley, R. A. 1997. Behavioral inhibition, sustained attention, and executive functions. Constructing a unifying theory of ADHD. Psychological Bulletin, 121, 65-94.

Bishop, D.V. M. 1997. Uncommon understanding. Development and disorders of language comprehension in children. Hove, United Kingdom: Psychology Press.

Bishop, D. V. M., \& Norbury, C. F. 2005. Executive functions in children with communication impairments, in relation to autistic symptomatology 2: Response inhibition. Autism, 9, 29-43.

Bjorklund, D. F., \& Harnishfeger, K. K. 1990. The resources construct in cognitive development: Diverse sources of evidence and a theory of inefficient inhibition. Developmental Review, 10, 48-71.

Brown, L., Sherbenon, R., \& Johnsen, S. K. 1997. Test of nonverbal intelligence. Third edition (TONI-3). Austin, Texas: pro-ed.

Copland, D. A., Chenery, H. J., \& Murdoch, B. E. 2000. Processing lexical ambiguities in word triplets: evidence of lexical-semantic deficits following dominant nonthalamic subcortical lesions. 
Neuropsychology, 14, 379-390.

Cornoldi, C., Marzocchi, G. M., Belotti, M., Caroli, M. G., De Meo, T., \& Braga, C. 2001. Working memory interference control deficit in children referred by teachers for ADHD symptoms. Child Neuropsychology, 7, 230-240.

Dunn, L. M., \& Dunn, L. M. 1997. Peabody Picture Vocabulary Test-III. American Guidance Service, Circle Pines, MN.

Ellis Weismer, S., \& Evans, J. 2002. The role of processing limitations in early identification of specific language impairment. Topics in Language Disorders, 22, 15-29.

Ellis-Weismer, S., Evans, J., \& Hesketh, L. J. 1999. An examination of verbal working memory capacity in children with specific language impairment. Journal of Speech, Language, and Hearing Research, 42, 1249-1260.

Friedman, N. P., \& Miyake, A. 2004. The relations among inhibition and interference control functions: A latent variable analysis. Journal of Experimental Psychology: General, 133, 101-135.

Gernsbacher, M. A., \& Faust, M. E. 1991. The mechanism of suppression: A component of general comprehension skill. Journal of Experimental Psychology: Learning, Memory, and Cognition, 17, 245-262.

Gillam, R. B., Cowan, N., \& Day, L. S. 1995. Sequential memory in children with and without language impairment. Journal of Speech and Hearing Research, 38, 393-402.

Hall, W. S., Nagy, W. E., \& Linn, R. 1984. Spoken words: Effects of situation and social group on oral word usage and frequency. Hillsdale, NJ: Erlbaum.

Hasher, L., Lustig, C., \& Zacks, R. T. 2007. Inhibitory mechanisms and the control of attention. In A. Conway, C. Jarrold, M. Kane, A. Miyake, \& J. Towse (Eds.). Variation in working memory. Oxford University Press.

Johannes, S., Wieringa, B. M., Mantey, M., Nager, W., Rada, D., Müller-Vahl, K. R., Emrich, H. M., Dengler, R., Münte, T. F., \& Dietrich, D. 2001. Altered inhibition of motor responses in Tourette Syndrome and Obsessive-Compulsive Disorder. Acta Neurologica Scandinavica, 104, 36-43.

Leonard, L. B. 1998. Children with specific language impairment. Cambridge, Massachusetts: the MIT Press.

Liss, M., Fein, D., Allen, D., Dunn, M., Feinstein, C., Morris, R., Waterhouse, L., \& Rapin, I. 2001. Executive functioning in high-functioning children with autism. The Journal of Child Psychology and Psychiatry and Allied Disciplines, 42, 261-270.

Marton, K. 2006. Do nonword repetition errors in children with specific language impairment reflect a weakness in an unidentified skill specific to nonword repetition or a deficit in simultaneous processing? Applied Psycholinguistics, 27, 569-573.

Marton, K., \& Schwartz, R. G. 2003. Working memory capacity limitations and language processes in children with specific language impairment. Journal of Speech, Language, and Hearing Research, 46, 1138-1153.

Marton, K., Schwartz, R. G., Farkas, L., \& Katsnelson, V. 2006. The effect of sentence length and complexity on working memory performance in Hungarian children with specific language impairment (SLI): A cross-linguistic comparison. International Journal of Language and Communication Disorders, 41, 653-673.

Marton, K., Schwartz, R. G., Kelmenson, L., \& Pinkhasova, M. (in preparation). The relationship between working memory and the long-term lexicon in children with specific language impairment.

McCormack, T., Brown, G. D. A., \& Vousden, J. I. 2000. Children's serial recall errors: Implications for theories of short-term memory development. Journal of Experimental Child Psychology, 76, 222-252.

Melara, R. D., Rao, A., \& Tong, X. 2002. The duality of selection: Excitatory and inhibitory processes in auditory selective attention. Journal of Experimental Psychology, 28, 279-306.

Montgomery, J. W. 1995. Examination of phonological working memory in specifically language impaired children. Applied Psycholinguistics, 16, 335-378.

Montgomery, J. W. 2000. Verbal working memory and sentence comprehension in children with specific language impairment. Journal of Speech, Language, and Hearing Research, 43, 293-308.

Montgomery, J. W. 2003. Working memory and comprehension in children with specific language impairment: What we know so far. Journal of Communication Disorders, 36, 221-231. 
Noterdaeme, M., Amorosa, H., Mildenberger, K., Sitter, S., \& Minow, F. 2001. Evaluation of attention problems in children with autism and in children with specific language disorder. European Child \& Adolescent Psychiatry, 10, 58-66.

Semel, E., Wiig, E. H., \& Secord, W. A. 1995. Clinical Evaluation of Language Fundamentals: CELF-3 Screening Test. San Antonio, TX: The Psychological Corporation.

Semel, E., Wiig, E. H., \& Secord, W. A. 2003. Clinical Evaluation of Language Fundamentals. Fourth edition. (CELF-4). The Psychological Corporation.

Stedron, J. M., Sahni, S. D., \& Munakata, Y. 2005. Common mechanisms for working memory and attention: The case of perseveration with visible solutions. Journal of Cognitive Neuroscience, 17, 623-631.

Wegner, D. M. 1992. You can't always think what you want: problems in the suppression of unwanted thoughts. Advances in Experimental Social Psychology, 25, 193-225.

Wenzlaff, R. M., \& Wegner, D. M. 2000. Thought suppression. Annual Review of Psychology, 51, 59-91.

Wilson, S. P., \& Kipp, K. 1998. The development of efficient inhibition: Evidence from directed forgetting tasks. Developmental Review, 18, 86-123.

(Manuscript received October 31, 2006; Revision accepted March 31, 2007)

\section{Appendix}

Sample sentences from the listening span task.

1. We have a big house on top of the hill.

Question: What is on top of the hill?

2. The little bird whistled a song.

Question: What did the little bird do?

3. Sharon dances to the music.

Question: What does Sharon do?

4. The baseball players lost their game.

Question: Who lost the game?

Examples of different inhibition errors.

1. Immediate distractor interference:

Stimulus: We have a big house on top of the hill.

The baseball players lost their game.

Response (recall of the sentence-final word: House, game

2. Delayed distractor interference:

Stimulus - previous set: The little bird whistled a song.

Sharon dances to the music.

Stimulus - current set: George reads his book.

Beth took her sister's toys.

Response to the current set: Dances, book

3. Perseveration:

Stimulus: Joe called Jim from my house.

Response: House.

Next stimulus: The school guard helps the child.

Response: House. 